\title{
Elaboração de mapas conceituais como instrumento de aprendizado remoto de estudantes de medicina
}

\author{
Elaboration of conceptual maps as remote learning \\ instrument of medice students
}

Tauane Vechiato', Anielle Lima Martins Santos', Íngria Correia da Costa Morais Modesto', Giselle Menezes Gomes' ${ }^{1}$ Ana Mackartney de Souza Marinho'

'Programa de Pós-graduação em Medicina, Instituto Tocantinense Presidente Antônio Carlos, Palmas (TO), Brasil.

\section{RESUMO}

Objetivo: Nortear o desenvolvimento dos mapas conceituais através de um guia padronizado na forma de um passo a passo, com os tópicos mais encontrados nos estudos das patologias, permitindo a inclusão de informações relevantes ao estudo elencado. Método: Foi realizado um estudo de levantamento teórico com intuito de criar um passo a passo sobre mapas conceituais voltados as patologias médicas no contexto da metodologia PBL, utilizando ferramenta miMind ${ }^{T M}$, na disciplina de Sistemas Orgânicos Integrados da graduação em medicina. Resultado: Ao confrontar dois mapas conceituais, um elaborado sem critérios propostos e o outro a partir do método de passo a passo, verificou-se no último uma melhor harmonização, padronização, hierarquização, agilidade e sequência lógica. Conclusão: Pode-se inferir que os mapas conceituais padronizados, subsidiarão o conhecimento e pesquisa para atuais e futuros estudantes de medicina, assumindo uma fase ímpar do ensino, ressignificando e garantindo a eficácia do ensino ativo o qual se aplica o PBL.

PALAVRAS-CHAVE: Aprendizagem Baseada em Problemas. Educação à distância. COVID-19. Educação de Graduação em Medicina.

Recebido: mar, 11, 2021

Aceito: mai, 03, 2021

\section{COMO CITAR ESTE ARTIGO}

Vechiato T, Santos ALM, Modesto ICCM, Gomes GM, Marinho AMS.

Elaboração de mapas conceituais como instrumento de aprendizado como instrumen remoto de estudantes de medicina. Interdisciplinary Journal of Health Education. 1-7 https://doi. org/10.4322/ijhe.2020.015

\section{CORRESPONDÊNCIA}

Tauane Vechiato

Programa de Pós-graduação em Medicina, Instituto Tocantinense Presidente Antônio Carlos Quadra ARSE 32, Alameda 03, Lote 15, Bloco 01, apart. 203, Residencial Mirante Du Park, 306 Sul, Plano Diretor Sul, CEP 77021-034, Palmas (TO), Brasil

tauanevechiato@gmail.com

\section{FONTE DE FINANCIAMENTO}

Financiamento próprio.

\section{CONFLITO DE INTERESSE}

Os autores referem não haver

qualquer conflito de interesse.

O estudo foi realizado no Instituto

Tocantinense Presidente Antônio Carlos

(ITPAC/PALMAS), Palmas (TO), Brasil.

Todos os autores leram e aprovam

a versão final submetida ao

Interdisciplinary Journal of

Health Education (IJHE).

\section{ABSTRACT}

Objective: Guide the disinvolvement of the conceptual map through of padronized guide in a step by step form, with the most founded topics in the study of pathologies to grant inclusion of relevant information. Methods: Was realized a review of theoretical survey with intuit of make a step by step by conceptual maps turned for medical pathologies in the context of methodology $\mathrm{PBL}$, utilizing the instru-ment miMind ${ }^{\mathrm{TM}}$, in the discipline of Integrated Organic Systems in the graduation of medicine. Results: Comparing two conceptual maps, one elaborated without proposed criterions and the other one made by methodes step by step, was verified in the last one a better harmonization, padronization, hierarchy, agility and logical sequence. Conclusion: Was conclude that the padronized conceptual maps, will subsidize the knowledge and search for actuals and future medicine students, assuming a unic phase of ensine, reframing and securing the efficacy of the active teach that get applied the PBL.

KEYWoRDS: Problem-Based Learning. Education, Distance. COVID-19. Education, Medical, Undergraduate. 


\section{Introdução}

A Educação Médica no Brasil é comumente debatida e analisada por diversos estudiosos e instituições. As grades curriculares de diversas instituições de ensino da medicina permanecem burocráticas e de lenta modernização, dificultando por vezes o processo Ensino-pesquisa-comunidade ${ }^{1,2}$.

A complexidade dos processos de aprendizagem tem colocado enfoque na metodologia construtivista. O modelo PBL (Problem Based Learning) vem para romper com as metodologias tradicionais procurando se adaptar a novas tendências mundiais ${ }^{3}$

A aprendizagem baseada em problemas, conhecido por seu acrônimo em língua inglesa, PBL, é uma metodologia problematizadora fundada na Universidade McMasters, em meados de 1970 no Canadá pelo pesquisador e professor norteamericano Joseph Novak. Tem no construtivismo suas bases teóricas e se opõe ao método tradicional e passivo de transmissão de conhecimento ${ }^{3,4}$.

De acordo com a andragogia - ciência antiga que estuda a educação para adultos com a finalidade de buscar uma aprendizagem para o desenvolvimento de habilidades e conhecimento - o aluno na metodologia ativa tem autonomia para buscar informações, partindo do seu conhecimento prévio ${ }^{5}$. Deste modo, o aprendizado não se limita apenas à visão do professor, tornando-o mais crítico-reflexivo dos assuntos abordados $^{6,7}$.

Estudos apontam que uma das ferramentas na prática acadêmica que auxilia os estudantes de forma autônoma são os Mapas Conceituais tendo como objetivo representar o pensamento crítico utilizando a teoria de aprendizagem significativa de David Ausubel ${ }^{8-10}$.

O Mapa Conceitual é uma estratégia que possibilita o desenvolvimento de habilidades de diagnóstico de conceituações alternativas e uma ferramenta de ensino utilizada em diversas áreas do conhecimento e níveis educacionais. Este requer a capacidade de analisar, sintetizar, flexibilizar, organizar, além de identificar mecanismos de natureza hierárquica, conceitual e proposicional do conhecimento ${ }^{11}$. É também uma representação gráfica de significados, considerado uma ferramenta de organização do conhecimento que fornece um meio visual para representar possíveis relações entre conceitos e arranjo do conhecimento, que não puderam ser organizadas ou considerados por outros meios pedagógicos ${ }^{12,13}$.

Para a aplicação eficaz da metodologia ativa é necessário a utilização de ferramentas modernas e atualizadas, visto o constante desenvolvimento das tecnologias da informação. Dentro do rol de ferramentas e procedimentos se destacam a aplicação de estudos de caso, método de projetos (abordagem de problemas reais presenciados pelos alunos) e a sala de aula invertida. Atualmente ferramentas como Google Classroom ${ }^{\circledR}$ e Moodle estão entre as mais utilizadas para tal fim ${ }^{14}$.

No estado do Tocantins, Instituto Tocantinense Presidente Antônio Carlos - ITPAC / Palmas se destaca na implementação da metodologia de ensino ativa, possuindo no campus da capital Palmas curso de Medicina com sua metodologia PBL em seu projeto pedagógico de curso (PPC). Tal metodologia é observada principalmente na disciplina de Sistemas Orgânicos Integrados (SOI), que trabalha medicina baseada em problemas, onde o docente apresenta situações-problema nas aprendizagens de pequenos grupos (APG) com objetivo de gerar um pensamento crítico - reflexivo ${ }^{15}$.

No início do ano de 2020, com a declaração da pandemia causada pelo Sars-Cov-2 pela Organização Mundial de Saúde (OMS) e a implementação de protocolos de distanciamento e isolamento social, a faculdade ITPAC/Palmas precisou realizar a transição das aulas presenciais para aulas virtuais, desenvolvendo e implementando o módulo REAR - Regime Especial de Aprendizagem Remota. Desta forma, os acadêmicos que antes criavam mapas conceituais dentro da sala de aula, normalmente 
em quadro branco, agora utilizaram softwares sugeridos pela faculdade como ferramentas para a criação coletiva.

Em tempos incomuns como é o caso da pandemia e com o cenário de ensino no modo REAR, as ferramentas implementadas exigem síntese, criatividade e elaboração pelo próprio aluno, estimulando assim, o pensamento crítico-reflexivo e mantendo-o como protagonista. O presente trabalho justifica-se pela necessidade de aprofundamento teórico para criação de um método sistemático para o desenvolvimento de mapas conceituais na metodologia ativa.

\section{Método}

Foi realizado um estudo sobre mapas conceituais a partir de fundamentação teórica para criação de um passo-a-passo, voltados a patologias médicas com ênfase no PBL. Por se tratar de uma abordagem teórica, não houve necessidade de submissão ao comitê de ética e pesquisa (CEP).

Durante a pandemia do coronavírus declarada pela OMS, a instituição estabeleceu uso de plataformas digitais e inserção de ferramenta de mapas conceituais a fim de manter suas atividades acadêmicas, na disciplina de Sistemas Orgânicos Integrados (SOI) nos pequenos grupos de aprendizagem (APG) com ensino remoto - REAR. Nessa disciplina, os alunos se deparam com assuntos que compreendem o processo saúde-doença, bem como discussões de casos clínicos.

A realização das aulas ocorreu através de Software de videoconferência Zoom Video Communications Inc, ofertado pela faculdade e para a criação de mapas conceituais foi utilizado o programa miMind ${ }^{\mathrm{TM}}$, disponível no endereço eletrônico CryptoBees ${ }^{16}$. Este possui diversas ferramentas, sendo uma multiplataforma personalizada, além de facilidades como exportação em PDF e imagem.

Com o objetivo de nortear o desenvolvimento dos mapas conceituais, foi criado um guia padronizado na forma de um passo-a-passo, com os tópicos mais encontrados nos estudos das patologias de forma a permitir a inclusão de informações relevantes.

Tais itens do passo-a-passo seguem descritos abaixo:

Passo 1 - Tema e Objetivo APG: Identifique os objetivos, tema central da APG e realize a pesquisa bibliográfica necessária para estudo do assunto. Escolha do software para criação dos mapas conceituais;

Passo 2 - A escolha do software: Observe os softwares indicados pela instituição de ensino e escolha o programa que será utilizado para criação dos mapas conceituais. Considere como principal critério de escolha a facilidade de utilização da plataforma.

Passo 3 - Tema Principal: utilizando o software para criação dos mapas mentais, insira o tema principal como o primeiro "nó".

Sugere-se para a padronização do Tema Principal, Fonte: Arial, Tamanho de fonte: 50; Negrito; Cor de fundo: Clara, a critério;

Passo 4 - Subtemas: De forma simétrica e hierárquica, com criação e distribuição como "nós filho" conectados ao tema principal, elencando os seguintes tópicos:

- Etiologia;

- Epidemiologia;

- Fisiopatologia;

- Clínica da Doença;

- Complicações da Doença;

- Fatores de risco; 
- Diagnóstico;

- Prevenção;

- Tratamento.

Utilize as seguintes padronizações de Fonte para os subtemas, podendo adicionar subtemas que julgar necessário. Sugestão: fonte Arial, Tamanho de fonte: 40; Cor de fundo: Clara, a critério.

Passo 5 - Itens: Insira os itens-chave conectados aos subtemas.

Após o estudo e a seleção dos itens-chave para inserção no mapa conceitual, insiraos como "nós-filhos" dos subtemas. Utilize as seguintes padronizações de Fonte para os itens: Fonte Arial, Tamanho de fonte: 35; Cor de fundo: Clara, a critério;

Passo 6 - Revisão e Bibliografia: Revise todo conteúdo conforme as fontes bibliográficas estudadas e insira estas fontes em lista simples no canto inferior direito do mapa conceitual.

Utilize as seguintes padronizações de Fonte para as fontes bibliográficas: Fonte Arial, Tamanho de fonte: 12; lista simples;

Ao término da elaboração dos mapas conceituais é realizada a exportação em formato de leitura padrão como PDF e enviado para avaliação do orientador e posterior discussão.

Tais sugestões rompem a inércia inicial de desenvolver o conteúdo a ser compreendido no desenho técnico do mapa.

\section{Resultado}

Considerando a organização original do ensino do curso de medicina no módulo PBL, as atividades teóricas e práticas eram realizadas presencialmente nos laboratórios e auditórios da universidade, sendo essas com salas de aula no formato de metodologias ativas/sala invertida e intercambiáveis para a disposição física tradicional, assim o aluno possuía a liberdade para criar seu próprio método de estudo. Com a nova realidade, a faculdade disponibilizou softwares de reuniões virtuais, gerenciamento de ensino virtual e realizou uma adaptação na modalidade de encontros que naquele momento se tornou exclusivamente virtual.

As APG's deixaram as estruturas físicas da faculdade e passaram a ser realizadas pela ferramenta de reuniões virtuais. Anteriormente à pandemia, os alunos dispunham de estrutura como salas de aula exclusiva para as reuniões dos grupos munidos de equipamento de projeção digital, quadros brancos com escrita livre, sistema de condicionamento de ar e o suporte pontual do mediador ou orientador. Como ferramenta e requisito básico do processo de aprendizagem, os componentes dos grupos confeccionavam um mapa conceitual sobre o desenvolvimento do conhecimento do problema proposto.

Ao iniciar o modo remoto foram encontradas pequenas limitações para o desenvolvimento dos mapas conceituais, entre elas: dificuldade técnica na manipulação dos programas para sua execução e alinhamento do raciocínio na criação do conhecimento comum.

Anteriormente ao estudo do passo-a-passo, verificou-se algumas características técnicas importantes sobre o tema proposto que estão ausentes no mapa (Figura 1), tornando-o não padronizado e incompleto para o estudo, uma vez que havia dificuldades de sumarizar o assunto proposto

Observa-se na Figura 1 as características supracitadas de mapas criados inicialmente.

Com a aplicação de padrões de utilização do programa miMind ${ }^{\mathrm{TM}}$, como: fontes, tamanhos de letra de títulos e subtítulos e referência bibliográfica completa, obtêmse mapas conceituais mais harmônicos e padronizados, resultando em maior 


\section{ighe}

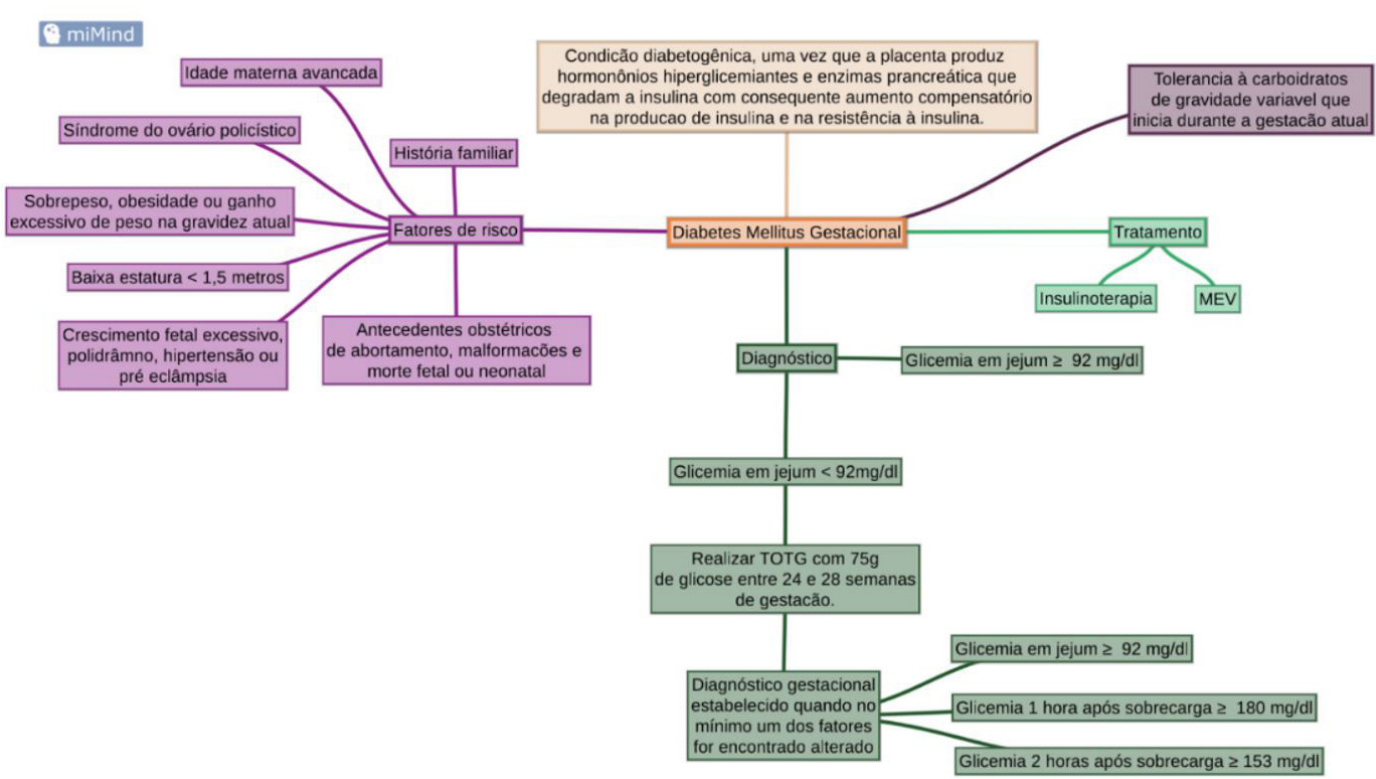

Figura 1. Mapa conceitual sobre o tema Diabetes Mellitus Gestacional sem a utilização do método elaborado pelos autores.

aproveitamento do conteúdo em estudos futuros, "organização" mental mais efetiva, além de melhor desempenho avaliativo no mapa.

O seguinte mapa (Figura 2) exemplifica a utilização do passo-a-passo proposto pelo artigo:

\section{Discussão}

Mapas conceituais são utilizados no ensino superior com resultados positivos nos últimos anos, foram observados um maior número de trabalhos sobre o tema em áreas diversificadas, porém não há muitos artigos em educação médica principalmente no âmbito do ensino remoto.

Os autores analisaram que o mapa da Figura 2 o qual utilizou os tópicos estabelecidos apresentou maior organização, estruturação, forma esquematizada e resolução dos problemas abordados, corroborando com um estudo de Mendonça ${ }^{17}$.

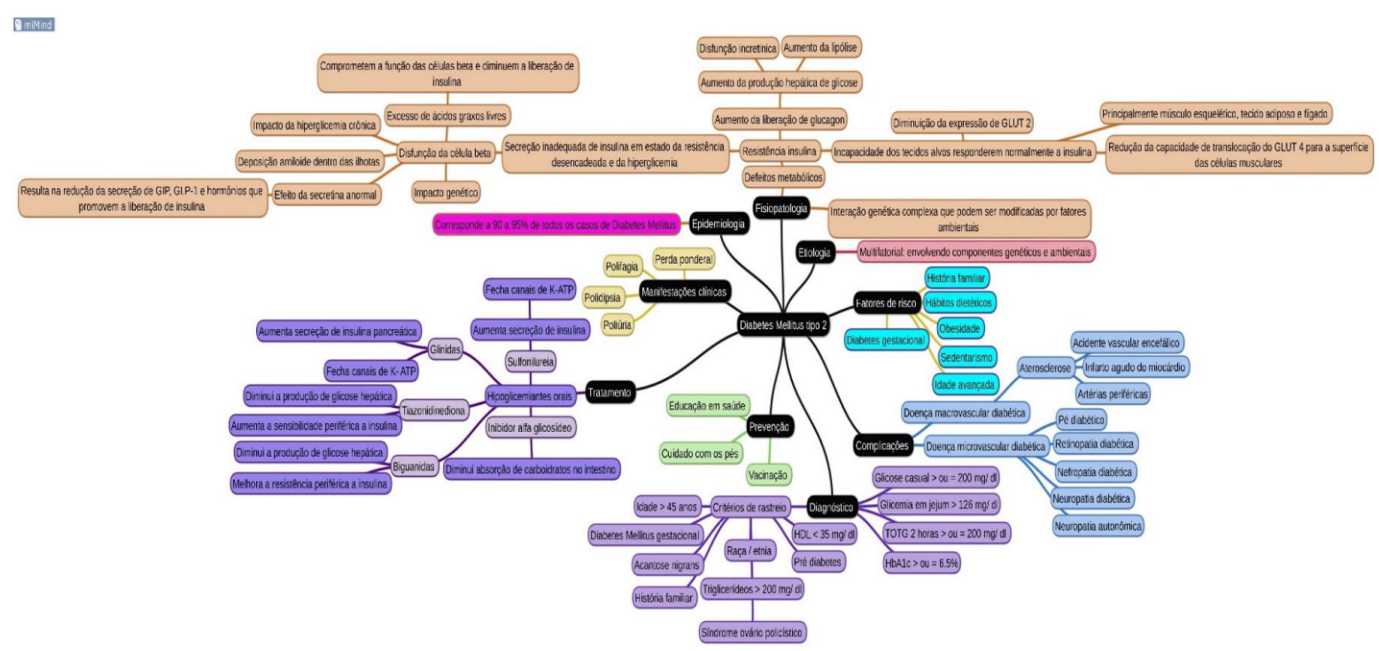

Figura 2. Mapa conceitual com o tema Diabetes Mellitus Tipo $2 \mathrm{com}$ a utilização do método proposto pelos autores. 
Carvalho et al. ${ }^{18}$ contempla em seu estudo que os mapas conceituais auxiliam os estudantes a organizar e integrar informações, em contrapartida problemas pontuais e recorrentes foram notados na confecção dos mesmos pelos alunos, como ausência de padrão organizacional e a falta de abordagem de todo conteúdo a ser explorado.

Os mapas conceituais ganharam destaque durante a implementação no módulo REAR se tornando a principal ferramenta de aprendizado durante a pandemia ${ }^{18}$.

Um levantamento teórico foi realizado sobre uma ferramenta utilizada na metodologia ativa e $\mathrm{PBL}^{2}$, alinhado ao atual cenário de pandemia e assim proposto um passo-a-passo para padronizar e nortear a criação dos mapas, considerando os principais subtemas abordados no conteúdo a ser explorado.

O método de passo-a-passo elaborado abordando temas e subtemas prédeterminados, favoreceu a harmonização, padronização, hierarquização, agilidade e sequência lógica dos assuntos propostos para criação dos mapas, permitindo melhor desempenho educacional dos acadêmicos estando de acordo com Moreira ${ }^{19}$.

Embasado em estudos de Maia et al. ${ }^{2}$ os mapas conceituais ajudam a organizar os pensamentos individuais e integrar informações, no modelo PBL e ensino remoto sendo assim compatível com nossos achados ${ }^{18,19}$.

\section{Conclusão}

De tal importância, podemos inferir que os mapas conceituais padronizados, nortearão o conhecimento e pesquisa para atuais e futuros estudantes de medicina, assumindo uma fase ímpar do ensino, ressignificando e garantindo a eficácia do ensino ativo o qual se aplica o PBL.

\section{Referências}

1. Machado CT, Carvalho AA. Os efeitos dos mapas conceituais na aprendizagem dos estudantes universitários. Educ Temát Digital. 2019;21(1):259-77. http://dx.doi.org/10.20396/etd.v21i1.8652010.

2. Maia MAG, Feitosa PWG, Neto MLR. Aprendizagem baseada em problemas na educação médica brasileira: uma revisão sistemática da literatura. Interfaces [Internet]. 2020 [citado em 2020 Ago 2];8(2):571-80. Disponível em: https://interfaces. leaosampaio.edu.br/index.php/revista-interfaces/article/view/738/PDF

3. Sebold LF, Martins FE, Rosa R, Carraro TE, Martini JG, Kempfer SS. Metodologias ativas: uma inovação na disciplina de fundamentos para o cuidado profissional de enfermagem. Cogitare Enferm. 2010;15(4):753-6. http://dx.doi.org/10.5380/ ce.v15i4.20381.

4. Amorim LM, Gonçalves J Jr, Rolim-Neto ML. Ensaio sobre a educação médica brasileira frente às vantagens e desvantagens do problem based learning (PBL). Rev e-ciência. 2017;5(1):20-2. http://dx.doi.org/10.19095/rec.v5i1.195.

5. Bellan ZS. Andragogia em ação: como ensinar adultos sem se tornar marcante. Santa Bárbara d 'Oeste: Z3 Editora; 2019.

6. Nunes JS. O uso pedagógico dos mapas conceituais no contexto das novas tecnologias. Int J e-Collaboration. $2010 ; 1$.

7. Bobato TT, Coelho ICMM, Ribeiro ER. Aprendizagem baseada em problemas no currículo integrado para graduação em medicina: construção da autonomia acadêmica. Contexto. 2018;33(105):52-67. http://dx.doi.org/10.21527/2179-1309.2018.105.52-67.

8. Novak JD. Learning, creating and using knowledge: concept maps as facilitative tools in schools and corporations. 2nd ed. Oxford: Routledge; 2010. http://dx.doi.org/10.4324/9780203862001.

9. Novak JD, Gowin DB. Learning how to learn. 1st ed. Ithaca: Cambridge University Press; 1984. http://dx.doi.org/10.1017/ CBO9781139173469.

10. Sousa SO. Aprendizagem baseada em problemas (PBL-Problem-Based Learning): estratégia para o ensino e aprendizagem de algoritmos e conteúdo [dissertação]. Presidente Prudente: Universidade Estadual Paulista; 2010.

11. Barbosa JAG, de Matos SS. Uso do mapa conceitual como ferramenta de ensino-aprendizagem no desenvolvimento do Trabalho de Conclusão de Curso. Rev Docência Ens Sup [Internet]. 2018 [citado em 2020 Ago 2];8(2):140-55. Disponível em: https://periodicos.ufmg.br/index.php/rdes/article/view/2452

12. Novak JD, Cañas AJ. The theory underlying concept maps and how to construct and use them [Internet]. Stanford: Stanford University; 2006. (Technical Report IHMC CmapTools) [citado em 2020 Ago 2]. Disponível em: https://web.stanford.edu/ dept/SUSE/projects/ireport/articles/concept_maps/The\%20Theory\%20Underlying\%20Concept\%20Maps.pdf

13. Mousinho SH. A utilização dos mapas conceituais para a construção dos conceitos no processo de aprendizagem. EaD \& Tecnol Digitais Educ [Internet]. 2019 [citado em 2020 Ago 2];7(9). http://dx.doi.org/10.30612/eadtde.v7i9.9609.

14. Monfradini JR, Bernini DSD. Ensino híbrido e metodologias ativas como ferramentas no processo de ensino e aprendizagem. Rev Esfera Acad Hum [Internet]. 2018 [citado em 2020 Ago 2];3(1):125-136. Disponível em: https://multivix.edu.br/wpcontent/uploads/2018/10/revista-esfera-humanas-v03-n01-artigo07.pdf 


\section{ijhe}

15. Faculdade de Ciências Humanas, Econômicas e da Saúde. Plano de desenvolvimento institucional - PDI (2017 a 2021 ) [Internet]. Palmas: FAHESA/ITPAC PALMAS; 2017 [citado em 2020 Ago 2]. Disponível em: https://assets.itpacpalmas. com.br/arquivos/institucional/pdi-fahesa-itpac-palmas-08-05-2018-edux.pdf

16. CryptoBees. miMind ${ }^{\mathrm{TM}}$ [Internet]. 2020 [citado em 2020 Ago 2]. Disponível em: https://mimind.cryptobees.com/

17. Mendonça CAS, Silveira FPRDA. Teaching parasitology with concept maps in laboratory lessons for teacher education courses. Am J Educ Res [Internet]. 2016 [citado em 2020 Ago 2];4(3):254-63. Disponível em: http://pubs.sciepub.com/ education/4/3/5/index.html

18. Carvalho DPSRP, Vitor AF, Barichello E, Villar RLA, Pereira-Santos VE, Ferreira-Junior MA. Aplicação do mapa conceitual: resultados com diferentes métodos de ensino-aprendizagem. Aquichan. 2016;16(3):382-91. http://dx.doi.org/10.5294/ aqui.2016.16.3.9.

19. Moreira MA. Organizadores prévios e aprendizagem significativa. Rev Chil Educ Cient [Internet]. 2012 [citado em 2020 Ago 2];7(2):23-30. Disponível em: http://www.if.ufrgs.br/ moreira/ORGANIZADORESport.pdf

\section{Contribuição dos autores}

Tauane Vechiato, escreveu introdução e realizou a formatação do artigo. Anielle Lima Martins Santos, escreveu metodologia do artigo e ajudou na criação dos mapas conceituais. Íngria Correia da Costa Morais Modesto, escreveu desenvolvimento do artigo. Giselle Menezes Gomes, escreveu conclusão e realizou revisão do artigo. Ana Mackartney de Souza Marinho, orientou a criação do artigo. 